\title{
Valentina Ponzetto, Musset ou la nostalgie libertine
}

Genève, Droz, 2007, 401 p.

Maxime Prévost

Université d'Ottawa

Tout comme Lamartine et Vigny, Alfred de Musset est un auteur que boude la critique contemporaine. La belle monographie de Valentina Ponzetto sur l'ensemble de son œuvre (prose, théâtre et poésie mêlés) pourrait contribuer à le sortir du semi-oubli où il est enfoui. L'auteure entend liquider une fois pour toutes le "Musset des jeunes filles», ce "poète pleurnichard au cœur brisé » (p. 11), pour étudier les liens, nombreux et féconds, qui unissent son œuvre à celle des libertins du XvIII siècle, plus particulièrement Crébillon fils, Casanova, Louvet de Couvray, Sade, Vivant Denon, Choderlos de Laclos, auxquels on peut 
ajouter le Richardson de Clarissa Harlowe et le Lorenzo Da Ponte de Don Giovanni, entre autres. Il en résulte une étude lumineuse qui éclaire tant l'intertexte libertin à l'œuvre dans les écrits de Musset que les modalités d'appropriation de cet univers littéraire par l'auteur de Lorenzaccio, bien conscient d'habiter une époque qui n'est plus celle de Faublas et de Valmont.

La culture dix-huitiémiste de Valentina Ponzetto est considérable, et sa connaissance tant de l'œuvre de Musset que de sa critique semble sans faille; c'est dire que l'étude à laquelle nous avons affaire est écrite par une auteure qui, de manière manifeste, préfère le siècle des Lumières à celui de Hugo et trouve en Musset l'objet tout désigné pour étudier la sensibilité libertine d'un écrivain romantique («Une évidence saute aux yeux: le monde de Musset n'est pas celui des autres romantiques », p. 37). Les deux premiers chapitres de l'étude, s'inspirant notamment d'Henri Lafon ${ }^{1}$ et de Michel Delon ${ }^{2}$, s'attachent à la description des lieux libertins (espaces géographiques et décors d'intérieur), c'est-à-dire aux topoï des romans du XVIII siècle que Musset revisite dans son théâtre, ses récits et sa poésie. C'est ensuite le comportement des personnages qui retient notre attention: Valentina Ponzetto énumère en effet des «éléments de libertinage à l'usage des personnages de Musset»(p.147), s'amusant à cerner «cet indéfinissable "je ne sais quoi" de libertin qui distingue [ses] personnages» (p. 155). Le chapitre de loin le plus imposant

${ }^{1}$ Henri Lafon, Les Décors et les choses dans le roman français du dix-huitième siècle de Prévost à Sade, Oxford, The Voltaire Foundation, 1992.

2 Michel Delon, L'Invention du boudoir, Paris, Zulma, 1999. 
(couvrant près du tiers de l'ouvrage) concerne la séduction et le «discours libertin» (p.243), de manière à proposer une typologie des séductions («dangereuses et corruptrices», "ludiques et complices», "velléitaires et maladroites ») que l'on trouve à l'œuvre chez Musset.

L'étude se termine sur un court chapitre, dont l'objet est captivant, intitulé « Musset et son lecteur », chapitre dans lequel apparaît la question du décalage et de l'ironie, c'est-à-dire dans lequel est historiquement mise en contexte l'utilisation que peut faire un écrivain romantique de l'intertexte libertin. Par ses allusions constantes, tantôt ouvertes tantôt voilées, aux textes de Denon, Casanova et Crébillon, Musset rangerait de son côté « un lecteur complice, coopératif, qui imaginera ce qu'on lui a laissé deviner selon ses propres goûts et ses propres désirs » (p. 334). Il y aurait là une forme de paradoxe, si l'on accepte l'idée, maintes fois exposée en cours de route, qu'une immense distance « sépare l'écriture de Musset [...] du goût de son temps»(p.61). Pourtant, si Musset peut jouer de cet intertexte libertin comme d'un outil de séduction, c'est sans doute que celui-ci a des résonnances concrètes auprès du lectorat de la monarchie de Juillet, tout «étouffé par le moralisme et la censure » (p. 358) que soit celui-ci. Telle est la principale réserve qu'inspire cet ouvrage: outre le cas d'exception que serait Musset, la période romantique y est schématisée comme celle des «mièvreries sentimentales » : «À l'école de Laclos, de Crébillon et de leurs confrères, Musset a appris à tempérer les déluges romantiques d'adjectifs, de phrases et de rhétorique par la clarté, la concision, les ellipses et les allusions malicieuses typiques du XVIII siècle, notamment libertin. » (p. 358) Or, cet intertexte libertin est aussi présent 
chez Hugo, chez Gautier, chez Soulié, chez Baudelaire, pour ne nommer que ceux-ci, de sorte qu'il serait dommage de ne pas ouvrir la fascinante étude de Valentina Ponzetto à un horizon plus vaste que l'œuvre du seul Musset. De tels prolongements seraient utiles précisément en ceci qu'ils cesseraient de renvoyer dos à dos XVIII et XIXe siècles, de manière à en objectiver les filiations souterraines, trop souvent occultées par la critique.

On notera enfin que l'auteure a délibérément choisi de ne pas tenir compte de Gamiani ou deux nuits d'excès, «ce récit érotique publié dans l'anonymat en 1833 dont l'attribution à Musset, malgré un débat animé qui dure depuis le XIXe siècle, demeure incertaine » (p. 32). Voilà qui est sage dans le contexte d'une thèse de doctorat, mais on pourra regretter que Valentina Ponzetto n'ait pas pris le parti, pour la publication finale, de faire peser son avis dans la balance: elle nous convainc pourtant, tout au long de son étude, qu'elle est l'une des personnes les mieux habilitées à le faire. 